\title{
Hacia una Salud Pública Pluralista: El Caso de los Plaguicidas y la Salud Humana
}

\author{
Álvaro Javier Idrovo \\ Médico, Especialista en Higiene y Salud Ocupacional, M.Sc. Salud Pública, M. Sc. Salud \\ Ambiental, Candidato a Ph. D. (candidato) Epidemiología. Centro de Investigación en Salud \\ Poblacional, Instituto Nacional de Salud Pública. Cuernavaca, México. \\ E-mail: idrovoaj@hotmail.com; idrovoaj@yahoo.com.mx
}

Recibido 22 Marzo 2005/Enviado para Modificación 13 Agosto 2005/Aceptado 6 Septiembre 2005

\section{RESUMEN}

El método epidemiológico es considerado la forma estándar de estudiar los problemas de salud pública. Debido a la complejidad de los problemas sanitarios, en las últimas décadas algunos autores llamaron al uso de métodos cualitativos. En este ensayo se muestra que el uso complementario de métodos cuantitativos y cualitativos es conservador y limitado, por lo que se sugiere que la salud pública requiere utilizar diversas aproximaciones epistemológicas, y no solo conjugar métodos cuantitativos y cualitativos. El caso de los plaguicidas y los efectos en la salud humana es usado como ejemplo para mostrar los aportes y limitaciones de diferentes modelos teóricos en salud pública (sanitarista, biomédico, epidemiológico clásico, higienista-preventivo, ecologista, epidemiológico social, económico, material-histórico y neoconservador), así como para poner en evidencia la necesidad de usar diferentes aproximaciones epistemológicas. Se concluye que una aproximación integradora permitirá que la salud pública sea más pluralista y democrática.

Palabras Clave: Investigación cualitativa, conocimiento, salud pública, plaguicida (fuente: DeCS, BIREME).

\section{ABSTRACT}

Toward a pluralistic public health: the case of pesticides and human health

Suggesting that epidemiology should be employed as the standard means of studying public health problems. Some authors have called for the use of qualitative research methods during the last few decades due to the complexity of sanitary problems. The complementary use of quantitative and 
qualitative methods is conservative and limited, which is why it is suggested that public health should use diverse epistemological approaches and not just conjugate quantitative and qualitative methods. The case of pesticides and their effect on human health is used as an example to show different theoretical models' contribution towards and limitations on public health (i.e. sanitary, biomedical, classical epidemiological, hygienist-preventative, ecological, social-epidemiological, economic, material-historical and neoconservative) as well as expressing the need to use different epistemological approaches. An integrated approach will lead to more pluralist and democratic public health.

Key Words: Qualitative research, knowledge, public health, pesticide (source: $\mathrm{MeSH}, \mathrm{NLM}$ ).

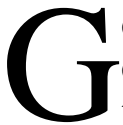
eneralmente se considera el método epidemiológico como la forma estándar para estudiar los problemas de salud pública (SP). Recientemente se menciona la necesidad de incluir métodos cualitativos para comprender la complejidad de estos problemas $(1,2)$. Gran parte del debate entre quienes argumentan la superioridad de los métodos cuantitativos y quienes defienden los cualitativos radica en la confusión entre diferencias metodológicas y epistemológicas; los "tradicionalistas" apoyan los métodos cuantitativos, mientras los "activistas" tienden por los métodos cualitativos (1).

Sin embargo, en la práctica el uso de uno u otro no implica necesariamente alguna adherencia política, por lo que un "tradicionalista" podría usar métodos cuantitativos y cualitativos, al igual que un "activista”. El llamado al uso complementario de métodos sigue siendo, sin embargo, conservador y limitado. Este ensayo muestra que la SP no sólo requiere el uso de ambos métodos, sino que necesita diferentes aproximaciones epistemológicas. Como ejemplo se utilizará un problema de salud ambiental, del que se listarán algunos estudios que muestran las fortalezas y debilidades de varios marcos conceptuales, con diferente enfoque epistemológico, utilizados en SP.

El problema de los potenciales efectos adversos sobre la salud humana de los plaguicidas usados en la agricultura tiene un sinnúmero de controversias, dificultades metodológicas y es de especial interés para los países del Tercer Mundo con actividades agrícolas y pecuarias $(3,4)$. Para los objetivos del presente artículo, se excluyeron del análisis aquellos casos en que los plaguicidas son usados para otras actividades, como la guerra y la erradicación de cultivos ilícitos, ya que pueden tener contextos sociales y políticos mucho más complejos que dificultan su análisis. Se partirá de considerar que en SP 
existen varios marcos conceptuales provenientes de diversos paradigmas (7) y se revisarán algunos ejemplos de los aportes de los marcos conceptuales sanitarista, biomédico, epidemiológico clásico, higienista-preventivo, ecologista, epidemiológico social, económico, sociomédico basado en el materialismo histórico y sociomédico neoconservador en el tema en cuestión. Las características de estos marcos conceptuales ya han sido analizadas $(8,9)$, y se encuentran resumidas en la Tabla 1.

\section{SALUD PÚBLICA Y PLAGUICIDAS}

La historia identifica dos momentos en relación con el año en que se sintetizó por primera vez el DDT: la anterior a 1874, caracterizada por el uso de pocas sustancias para lavar las plantas y protegerlas de las plagas, y la posterior a 1874, en la que se observa un crecimiento en el número de plaguicidas sintéticos (10). Primero fueron los insecticidas organoclorados, organofosforados y carbamatos, y posteriormente todo un amplio repertorio de sustancias con diferentes tipos de acción y plaga objetivo (11). El desarrollo de plaguicidas se debe a varios factores, entre los que sobresalen las condiciones biológicas (v.gr. resistencia de las plagas ya que muchas especies son capaces de desarrollar tolerancia en menor o mayor grado (12), así como las políticas de mercado. Además, los patrones de uso diferenciales entre los países guardan relación con las diferentes políticas comerciales y sanitarias.

\section{MARCOS CONCEPTUALES HEGEMÓNICOS}

Desde comienzos del siglo XX, varios marcos conceptuales han influido en la forma en que la SP entiende la relación entre plaguicidas y salud humana, así como las políticas sanitarias implantadas. Si bien es difícil encontrar ejemplos nítidos donde se manifieste un solo marco conceptual, a continuación se esquematizarán aportes y limitaciones de cada uno de éstos mediante algunos trabajos que tipifican las diferentes aproximaciones epistemológicas.

Marco conceptual sanitarista

El periodo de predominio de este marco conceptual se traslapa con el inicio del desarrollo de los plaguicidas sintéticos. Desde esta perspectiva, fueron muchos los efectos benéficos que los plaguicidas mostraron sobre la salud, sobresaliendo el control de la epidemia de tifo transmitida por piojos en Napolés en 1944 y las enfermedades transmitidas por vectores en todo el mundo (13), y en el control de las plagas de vegetales que son alimento para humanos. Desde esta aproximación los plaguicidas fueron aliados en el saneamiento de zonas endémicas de enfermedades. Sin embargo, este enfoque 
fue criticado debido a las denuncias en relación a su uso. La publicación de La primavera silenciosa en 1962 (14), en el que se hacen críticas al DDT, cambió la forma de pensar del público general frente a los plaguicidas. Posteriormente muchos otros libros e informes se han editado, como La conspiración plaguicida y El círculo del veneno, con diversos matices, que actualizaron de alguna manera los postulados de Carson.

Tabla 1. Principales características de algunos marcos conceptuales en salud pública, y aportes y limitaciones en relación al estudio de los plaguicidas y la salud humana

\begin{tabular}{|c|c|c|}
\hline \multirow{2}{*}{$\begin{array}{c}\text { Marco } \\
\text { conceptual }\end{array}$} & \multicolumn{2}{|c|}{ Estudio plaguicidas - salud humana } \\
\hline & Aportes & Limitaciones \\
\hline Sanitarista & $\begin{array}{l}\text { Evidencia los efectos benéficos de } \\
\text { los plaguicidas en el control de las } \\
\text { enfermedades transmitidas por vec- } \\
\text { tores. }\end{array}$ & $\begin{array}{l}\text { No es capaz de apreciar fácilmente } \\
\text { los potenciales efectos adversos } \\
\text { asociados con la exposición a los } \\
\text { plaguicidas. }\end{array}$ \\
\hline Biomédico & $\begin{array}{l}\text { Permite comprender los } \\
\text { mecanismos biológicos relacionados } \\
\text { con la acción y los efectos adversos } \\
\text { de los plaguicidas. }\end{array}$ & $\begin{array}{l}\text { Ignora los determinantes sociales } \\
\text { que condicionan la exposición a los } \\
\text { plaguicidas y las políticas re- } \\
\text { guladoras. }\end{array}$ \\
\hline $\begin{array}{l}\text { Epidemiológico } \\
\text { clásico }\end{array}$ & $\begin{array}{l}\text { Permite tener estimaciones de la } \\
\text { ocurrencia de intoxicaciones agudas } \\
\text { por plaguicidas, y explorar algunos } \\
\text { efectos por exposición crónica. }\end{array}$ & $\begin{array}{l}\text { Tiene grandes problemas en la } \\
\text { medición de la exposición, lo que ha } \\
\text { impedido llegar a conclusiones } \\
\text { contundentes. }\end{array}$ \\
\hline $\begin{array}{l}\text { Higienista } \\
\text { preventivo }\end{array}$ & $\begin{array}{l}\text { Enfatiza en el uso de elementos de } \\
\text { protección personal para disminuir la } \\
\text { exposición, lo que ha servido de } \\
\text { sustento para muchas políticas } \\
\text { preventivas en SP. }\end{array}$ & $\begin{array}{l}\text { No es crítico en relación al uso de } \\
\text { plaguicidas, y tiende a culpabilizar a } \\
\text { los individuos de la exposición al no } \\
\text { usar elementos de protección perso- } \\
\text { nal. }\end{array}$ \\
\hline Ecologista & $\begin{array}{l}\text { Permite una mejor comprensión del } \\
\text { ecosistema en el cual ocurre la } \\
\text { exposición y sus efectos, haciendo } \\
\text { énfasis en el ambiente físico. }\end{array}$ & $\begin{array}{l}\text { No es capaz de incorporar los } \\
\text { determinantes sociales de la ex- } \\
\text { posición a los plaguicidas. }\end{array}$ \\
\hline $\begin{array}{l}\text { Epidemiológico } \\
\text { social }\end{array}$ & $\begin{array}{l}\text { Enfatiza su análisis en los } \\
\text { macrodeterminantes de las exposi- } \\
\text { ciones y las condiciones de salud. }\end{array}$ & $\begin{array}{l}\text { Tiene problemas en la medición de } \\
\text { constructos sociales complejos. }\end{array}$ \\
\hline Económico & $\begin{array}{l}\text { Brinda una traducción económica a } \\
\text { los beneficios y riesgos asociados } \\
\text { con el uso de los plaguicidas. }\end{array}$ & $\begin{array}{l}\text { Prioriza el componente económico } \\
\text { sobre los demás determinantes; se } \\
\text { corre el riesgo de cuantificar } \\
\text { inadecuadamente algunos atributos } \\
\text { del individuo y las poblaciones. }\end{array}$ \\
\hline $\begin{array}{l}\text { Materialismo } \\
\text { histórico }\end{array}$ & $\begin{array}{l}\text { Enfatiza en el efecto que tienen las } \\
\text { formas de producción en la } \\
\text { exposición a los plaguicidas, dentro } \\
\text { de contextos socio-históricos } \\
\text { específicos. }\end{array}$ & $\begin{array}{l}\text { Tiende a ignorar las condiciones } \\
\text { biológicas e individuales que fa- } \\
\text { vorecen la ocurrencia de efectos } \\
\text { adversos. }\end{array}$ \\
\hline Neoconservador & $\begin{array}{l}\text { Ha permitido que la opinión de legos } \\
\text { se sume a la de los expertos, en la } \\
\text { decisión de políticas en SP. }\end{array}$ & $\begin{array}{l}\text { No puede aislar el efecto de los } \\
\text { factores que incluye en su análisis, } \\
\text { limitando así su ponderación. }\end{array}$ \\
\hline
\end{tabular}

Marco conceptual biomédico 
La medicina ha manejado los efectos de los plaguicidas. Sin embargo, con excepción del "síndrome intermedio", son pocos los aportes que la medicina ha brindado. Este síndrome ocurre después de una intoxicación aguda con algunos organofosforados y se caracteriza por parálisis de algunos músculos (15). Una ciencia que abordó el problema de los plaguicidas, apoyando la medicina, fue la toxicología. Con estudios in vitro e in vivo se han estudiado efectos agudos, subagudos y crónicos. Su importancia ha sido tal, que con base en sus métodos se ha construido el proceso de evaluación del riesgo (16), que consiste en la extrapolación de datos obtenidos de animales o humanos con intensa exposición a poblaciones en condiciones específicas, con menor exposición, usando estimadores del riesgo. El "método científico" está presente en este proceso ya que incluye un análisis caso por caso de todos los hechos, utilizando juicios de expertos y el criterio de la evidencia. Esta metodología es aplicada como medida preventiva a las nuevas sustancias antes de su comercialización por agencias internacionales.

\section{Marco conceptual epidemiológico clásico}

La epidemiología ha registrado las epidemias permitiendo tener una panorámica de la ocurrencia de intoxicaciones (17). Este fue el primer aporte que tuvo este marco conceptual, describiendo la ocurrencia en términos de persona, tiempo y lugar. Estudios más recientes han permitido identificar que las intoxicaciones no sólo se deben a exposiciones ocupacionales, sino que pueden ocurrir en condiciones diferentes, como el suicidio y el intento suicida (18), y mediante el "enfoque de riesgo" identificar a los grupos con mayor probabilidad de intoxicación (19). Muchos otros eventos han sido estudiados con las técnicas epidemiológicas; sin embargo, en esta búsqueda aún muchos de los interrogantes se mantienen debido a las dificultades que acarrea su investigación. Por ejemplo al estudiar el DDT, la evidencia epidemiológica sobre efectos reproductivos y cáncer de mama presenta resultados no consistentes. En relación con otros efectos endocrinos, sólo la inhibición tiroidea por el Amitrole y el Mancozeb han sido observados tanto en animales como en humanos (20).

\section{Marco conceptual higienista preventivo}

Este marco conceptual ha tenido gran aceptación en SP, influyendo en muchas políticas de manejo seguro de plaguicidas. Por ejemplo, un estudio con métodos cuantitativos y cualitativos pudo establecer que, pese a que los trabajadores tienen conocimiento de los efectos por la exposición, no usan elementos de protección debido a que no los consideran adecuados a sus condiciones climáticas y socioeconómicas (21). Resultados como estos se han re- 
portado en todo el mundo. Este enfoque no presenta críticas al uso de los plaguicidas, y parte de que el objetivo de la SP es minimizar la exposición. Por esto ha sido fuertemente criticado ya que tiende a "culpabilizar" del no uso de elementos de protección a los individuos expuestos olvidando el contexto en que ocurre dicha intoxicación.

\section{Marco conceptual ecologista}

Este marco requiere una comprensión más integral del ecosistema en el que ocurre la exposición y el efecto adverso, para determinar donde se pierde el equilibro entre éstos. En un estudio hecho en zonas productoras de papas en Ecuador (un agroecosistema localizado entre los 2400 y 3500 m.s.n.m., con pasto para el consumo de bovinos, y donde las enfermedades causadas por Phytophtera infestans y Premnotrypes vorax son las principales amenazas para la producción) se observaron intoxicaciones agudas, que los plaguicidas tienen un efecto benéfico sobre los cultivos, y que los campesinos no los usan irracionalmente, de manera similar a otros estudios de efectividad y en contra de las creencias generales (22). Dentro de este mismo enfoque se encuentran varios estudios sobre el control de insectos vectores de enfermedades (23). En todos estos el conocimiento del ecosistema fue el objetivo o permitió realizar algunas intervenciones con el fin de disminuir la ocurrencia de enfermedades, siendo sus resultados no consistentes en todos los casos.

Los anteriores marcos conceptuales han brindado aportes al conocimiento, especialmente para efectos agudos y sub-agudos. Sin embargo tienen limitaciones como el desarrollo precario de métodos para estudiar las mezclas de plaguicidas, las cuales son las formas habituales de uso y las dificultades para explorar efectos por exposición crónica a bajas concentraciones. Hay más de mil plaguicidas y sólo menos de 50 métodos para su cuantificación (24). Por otra parte, los largos periodos de latencia de los eventos crónicos y la precaria medición de los plaguicidas, han dificultado enormemente el estudio de los efectos tóxicos a largo plazo.

\section{MARCOS CONCEPTUALES EMERGENTES}

Más recientemente han aparecido algunos aportes de las disciplinas sociales para entender el contexto en el que se usan los plaguicidas, así como su potencial efecto sobre la salud. Sin embargo, aún son escasos los estudios que abordan temas ambientales (25). A continuación se presentan algunos ejemplos que permiten mostrar su utilidad. 
Marco conceptual epidemiológico social

Con este término se entienden varias corrientes de pensamiento en SP; éstas son: i) la psicosocial, ii) la producción social de la enfermedad o economía política de la salud, y iii) la ecosocial con sus modelos de niveles múltiples (26). Para este ensayo se consideró como "marco conceptual epidemiológico social" al primero y al tercero; el otro marco conceptual se fundamenta en el materialismo histórico y será revisado más adelante. Este marco conceptual enfatiza en los factores socioeconómicos como determinantes de la exposición a plaguicidas y de la ocurrencia de efectos adversos en la salud. Un estudio realizado en Brasil sirve para ejemplificarla (27), ya que en él se midió la actividad de las enzimas colinesterasas entre agricultores expuestos a plaguicidas, observando una asociación con el nivel educativo y condiciones relacionadas con el trabajo.

\section{Marcos conceptuales económicos}

Este marco incorpora las múltiples aproximaciones propias de la economía. Los más clásicos estiman los costos de usar plaguicidas y la carga económica de las intoxicaciones $(28,29)$. Otros estudios señalan los "costos ocultos” del uso de plaguicidas; por ejemplo, una investigación en Zimbabwe identificó que un alto porcentaje de los beneficios económicos por usar plaguicidas deben invertirse en el tratamiento de intoxicados, concluyendo que en algunos casos los gastos netos por tratamiento exceden los beneficios económicos de usar los plaguicidas (30).

Aproximación desde el materialismo histórico

La importancia de entender el contexto socio-histórico ha sido evidenciada en diversos estudios. En la agricultura sudafricana ha existido un paternalismo en la relación empleado-empleador, expresado en condiciones de vida austeras, prácticas de trabajo coercitivas y violencia contra los trabajadores. Allí el pago del trabajo se hace con bebidas con bajo contenido alcohólico, el cual juega un papel central en la selección, retención y reproducción del trabajo, constituyendo un elemento de control social. Se postula que el alcoholismo predispone a los trabajadores a intoxicaciones, debido a la desinhibición, descuido y conducta propensa a los accidentes; además, la intoxicación por alcohol podría enmascarar los efectos de los plaguicidas (31).

A finales de los 70, se describió que las empresas manufactureras con tecnologías peligrosas y procesos químicos se trasladaron hacia los países en desarrollo, evitando la compensación por enfermedades ocupacionales y 
ambientales asociadas con dichos procesos. Esta exportación de "industrias asesinas” hacia países con poca o ninguna regulación es consecuencia de las políticas globales de mercado (32). Con base en estos argumentos, en El círculo del veneno se llama la atención del público, al describir que los plaguicidas producidos en Norteamérica son exportados hacia países del Tercer Mundo, donde son usados para fumigar alimentos, que luego son consumidos por norteamericanos. En respuesta a este "círculo", los agricultores mexicanos adoptaron nuevos plaguicidas que cumplieran los requisitos estadounidenses, dejando de usar los prohibidos en Norteamérica, y configurando una "espiral del veneno" al ocurrir un doble paso a través de los límites nacionales debido a la dependencia de la agricultura en los plaguicidas y las tecnologías relacionadas (33). Estos argumentos han sido usados para describir el problema de las intoxicaciones. Uno de los casos más lamentables ocurrió con cultivadores de banano en Costa Rica, quienes se exponían al DBCP. En estas condiciones muchos trabajadores se volvieron infértiles, pese a que años antes el DBCP había sido prohibido en los Estados Unidos de América (EUA) al observarse estos efectos entre productores (34).

\section{Aproximación neoconservadora}

Este marco se ha consolidado como el complementario a los enfoques hegemónicos (1). Uno de los aportes son las actividades de manejo del riesgo, que complementan la evaluación de riesgo, y basándose en sus resultados propicia una fase de negociación entre los actores sociales involucrados. Este elaborado procedimiento tiene muchas críticas en relación con problemas de justicia ambiental; el racismo y el poder económico y social son algunas de las barreras ha superar, pero que en la práctica real no frecuentemente se hace (35). Con esta aproximación hay estudios que exploran la percepción del riesgo, y que pueden ayudar a definir las políticas sanitarias. Por ejemplo, en EUA se ha incrementado la preocupación de grupos de no campesinos en relación con los plaguicidas presentes en agua y alimentos. Sin embargo, existe una percepción del riesgo muy diferente entre los campesinos que usan agroquímicos; entre éstos, la percepción de efectos es mucho menor y está condicionada por las implicaciones económicas que acarrea la decisión de su uso (36). Otro estudio evidencia las limitaciones de la epidemiología usando un caso de exposición a mezclas de plaguicidas y la ocurrencia de malformaciones congénitas; allí se afirma que por abolir los resultados falsos positivos, se concluye que la "casualidad" es la "causa" de muchos eventos en salud (37). Estos son ejemplos de la "epidemiología popular” (25), en la que la población detecta y actúa sobre peligros ambientales y enfermedades. Un estudio que ha mostrado las potencialidades de los métodos cualitativos es el realizado con niños indígenas que se exponen a mez- 
clas de plaguicidas. Utilizando métodos cuantitativos y cualitativos fue posible identificar que los expuestos tienen efectos neuroconductuales (38).

\section{HACIA UNA SALUD PUBLICA DEMOCRÁTICA Y PLURALISTA}

En este ensayo se revisaron unos estudios que ejemplifican las potencialidades de algunos marcos conceptuales que actúan en la SP. Lo aportado por un marco conceptual, generalmente, puede no ser captado por los otros. Se puede concluir que para problemas complejos, como el aquí presentado, se requieren estudios complejos y aproximaciones con diferentes marcos conceptuales. Ya que cada vez se insiste más en la necesidad de conjugar métodos cualitativos con cuantitativos $(1,2)$, considero que el problema no es tan simple. Una comprensión holística del proceso salud-enfermedad en humanos es necesario aceptar aproximaciones aun con diferente enfoque epistemológico. El reconocer que los problemas sanitarios no pueden ser entendidos por una sola mirada es el primer paso para forjar una SP democrática y pluralista. Pero, ¿cómo podrían integrarse éstos en un marco conceptual? No hay una respuesta y lo que existe, hasta ahora, no son más que propuestas. La denominada "epidemiología política" (39) es una propuesta que sin transgredir los métodos epidemiológicos, intenta entender el impacto que los procesos políticos y burocráticos tienen sobre la información generada. Otra propuesta es la aproximación ecosistémica, que utiliza metodologías participativas sensibles a los determinantes sociales y de género de manera transdisciplinaria (40); ésta ha dado aportes tanto de conocimiento como en la solución de problemas sanitarios, pero puede ser difícil de implantar en todos los problemas de SP. Sea cual sea la aproximación adoptada, lo importante es que en la nueva propuesta la SP asuma que "la verdad" puede ser buscada mediante el fomento cooperativo de todos los marcos conceptuales. Este llamado a una nueva aproximación integradora se fundamenta en que optar por un único modelo conceptual sólo sería adecuado si éste fuese superior a los demás, al proveer una mejor explicación de la evidencia (41). El caso aquí analizado evidencia que este no es el caso, y que para tener una mejor comprensión del problema se requiere tener varias visiones, obtenidas mediante métodos cuantitativos y cualitativos, pero, aún más importante, provenientes de diferentes aproximaciones epistemológicas. La nueva propuesta no debería flexibilizar su "objetividad" y desechar sus métodos (42). El objetivo será aunar los esfuerzos de todas las aproximaciones en procura del conocimiento, y tener herramientas más adecuadas para prevenir la enfermedad y promover la salud. Mientras esto se concreta una opción alterna es aceptar explicaciones diferentes, y no quedarse exclusivamente con la limitada aproximación de los modelos hegemónicos • 


\section{REFERENCIAS}

1. Baum F. Researching public health: Behind the qualitative-quantitative methodological debate. Soc Sci Med 1995;40:459-468.

2. Brown P. Qualitative methods in environmental health research. Environ Health Perspect 2003;111:1789-1798.

3. Jeyaratnam J. Health problems of pesticide usage in the Third World. Br J Ind Med 1985;42:505-506.

4. Wesseling C. McConnell R, Partanen T, Hogstedt C. Agricultural pesticide use in developing countries: Health effects and research needs. Int J Health Serv 1997;27:273-308.

5. Frenk J. The new public health. Annu Rev Public Health 1993;14:469-490.

8. Arredondo A. Análisis y reflexión sobre modelos teóricos del proceso salud-enfermedad. Cad Saude Publica 1992;8:254-261.

9. Arredondo A. Marcos conceptuales en salud pública: modelos, paradigmas o propuestas disciplinarias. Cuad Med Soc (Chile) 1993;34:29-36.

10. Cassida JE, Quistad GB. Golden age of insecticide research: Past, present, or future? Annu Rev Entomol 1998;43:1-16.

11. Idrovo AJ. Vigilancia de las intoxicaciones con plaguicidas en Colombia. Rev Salud Pública (Bogotá) 2000;2:36-46.

12. Georghiou GP. The evolution of resistance to pesticides. Annu Rev Ecol System 1972;3:133-168.

13. Packard RM. "No other choice": Global malaria eradication and the politics of international health in the post-war era. Parassitologia 1998;40:217-229.

14. Carson R. Silent spring. Boston: Houghton Mifflin; 1962.

15. Senanayake N, Karalliedde L. Neurotoxic effects of organophosphorus insecticides. An intermediate syndrome. N Engl J Med 1987;316:761-763.

16. Barnard RC. Scientific method and risk assessment. Regul Toxicol Pharmacol 1994;19:211-218.

17. Idrovo AJ. Intoxicaciones masivas con plaguicidas en Colombia. Biomédica 1999;19:67-76.

18. Van der Hoek W, Konradsen F, Athukorala K, Wanigadewa T. Pesticide poisoning: A major health problem in Sri Lanka. Soc Sci Med 1998;46:495504.

19. Wesseling C, Castillo L, Elinder CG. Pesticide poisoning in Costa Rica. Scand J Work Environ Health 1993;19:227-235.

20. Cocco P. On the rumors about the silent spring. Review of the scientific evidence linking occupational and environmental pesticide exposure to endocrine disruption health effects. Cad Saúde Pública 2002;18:379-402.

21. Sivayoganathan C, Gnanachandran S, Lewis J, Fernando M. Protective measure use and symptoms among agropesticide applicators in Sri Lanka. Soc Sci Med 1995;40:431-436.

22. Crissman CC, Cole DC, Carpio F. Pesticide use and farm worker health in Ecuadorian potato production. Am J Agr Econ 1994;76:593-597. 
23. MacCormack CP. Human ecology and behaviour in malaria control in tropical Africa. Bull World Health Organ 1984;62(Suppl):81-87.

24. He F. Biological monitoring of occupational pesticides exposure. Int Arch Occup Environ Health 1993;65(Suppl.):S69-S76.

25. Brown P. Popular epidemiology and toxic waste contamination: Lay and professional ways of knowing. J Health Soc Behav 1992;33:267-281.

26. Krieger N. Theories for social epidemiology in the 21st century: An ecosocial perspective. Int J Epidemiol 2001;30:668-677.

27. Oliveira-Silva JJ, Alves SR, Meyer A, Perez F, Sarcinelli PN, da Costa Mattos RC, Moreira JC. Influência de fatores socioeconômicos na contaminação por agrotóxicos, Brasil. Rev Saude Publica 2001;35:130-135.

28. Soares W, Moro S, Almeida RM. Rural workers' health and productivity: An economic assessment of pesticide use in Minas Gerais, Brazil. Appl Health Econ Health Policy 2002;1:157-164.

29. Cole DC, Carpio F, León N. Economic burden of illness from pesticide poisonings in highland Ecuador. Rev Panam Salud Publica 2000;8:196-201.

30. Maumbe BM, Swinton SM. Hidden health costs of pesticide use in Zimbabwe's smallholder cotton growers. Soc Sci Med 2003;57:1559-1571.

31. London L. The 'dop' system, alcohol abuse and social control amongst farm workers in South Africa: A public health challenge. Soc Sci Med 1999;48:1407-1414.

32. Castleman BI. The export of hazardous factories to developing nations. Int J Health Serv 1979;9:569-606.

33. Wright A. Rethinking the circle of poisoning. The politics of pesticide poisoning among Mexican farm workers. Latin Am Perspect 1986;51:26-59.

34. Sass R. Agricultural "killing fields": The poisoning of Costa Rican banana workers. Int J Health Serv 2000;30:491-514.

35. Corburn J. Environmental justice, local knowledge, and risk: The discourse of a community-based cumulative exposure assessment. Environ Manage 2002;29:451-466.

36. Tucker M, Napier TL. Perceptions of risk associated with use of farm chemicals: Implications for conservation initiatives. Environ Manage 1998;22:575587.

37. Lloyd M. Analysis on the move: Deconstructing troublesome health questions and troubling epidemiology. Qual Health Res 2000;10:149-163.

38. Guillette EA, Meza MM, Aquilar MG, Soto AD, Garcia IE. An anthropological approach to the evaluation of preschool children exposed to pesticides in Mexico. Environ Health Perspect. 1998; 106:347-353.

39. Brownlea A. From public health to political epidemiology. Soc Sci Med 1981;15D:57-67.

40. Forget G, Lebel J. An ecosystem approach to human health. Int J Occup Environ Health. 2001; 7(suppl.1):S3-SS36.

41. Martin B. Dissent and heresy in medicine: Models, methods, and strategies. Soc Sci Med 2004;58:713-725.

42. Gori GB. Epidemiology and public health: Is a new paradigm needed or a new ethic? J Clin Epidemiol. 1998; 51:637-641. 